\title{
THE EXISTENCE AND EXPONENTIAL BEHAVIOR OF SOLUTIONS TO STOCHASTIC DELAY EVOLUTION EQUATIONS WITH A FRACTIONAL BROWNIAN MOTION
}

\author{
T. CARABALLO, M. J. GARRIDO-ATIENZA, AND T. TANIGUCHI
}

\begin{abstract}
In this paper we investigate the existence, uniqueness and exponential asymptotic behavior of mild solutions to stochastic delay evolution equations perturbed by a fractional Brownian motion $B_{Q}^{H}(t)$ :

$$
d X(t)=\left(A X(t)+f\left(t, X_{t}\right)\right) d t+g(t) d B_{Q}^{H}(t),
$$

with Hurst parameter $H \in(1 / 2,1)$. We also consider the existence of weak solutions.
\end{abstract}

\section{INTRODUCTION}

Fractional Brownian motions (fBm) appear naturally in the modelling of many complex phenomena in applications when the systems are subject to "rough" external forcing. An $\mathrm{fBm}$ is a stochastic process which differs significantly from the standard Brownian motion and semi-martingales, and others classically used in the theory of stochastic processes. As a centered Gaussian process, it is characterized by the stationarity of its increments and a medium- or long-memory property. It also exhibits power scaling with exponent $H$. Its paths are Hölder continuous of any order $H^{\prime} \in(0, H)$. When $H=1 / 2$ the $\mathrm{fBm}$ becomes the standard Brownian motion. However, when $H \neq 1 / 2$, the $\mathrm{fBm} B^{H}$ behaves in a completely different way than the standard Brownian motion; in particular, neither is a semi-martingale nor a Markov process.

From the point of view of the classical theory, the most obvious problem with $\mathrm{fBm}$ is precisely the lack of the martingale and Markov properties. The former prevents the use of a well-established integration theory. The latter means that there is no direct connection between $\mathrm{fBm}$ and differential operators.

The lack of the martingale property is a main mathematical challenge: when switching from Brownian motion $B^{1 / 2}$ to $\mathrm{fBm} B^{H}$, how does one define a proper notion of stochastic integral? There are three main integration techniques, two of which are trajectorial in nature, with some random component (Russo-Vallois or other regularizations/discretizations, and Rough Path theory), and the third which is entirely stochastic (Skorohod integral based on the Malliavin calculus). These techniques have one common point: they get harder as $H$ gets smaller; the more the paths of the stochastic process are irregular, the harder it is to integrate against them. Some of the most famous outstanding questions in stochastic analysis today are tied to this issue. This identifies path regularity as a key benchmark to evaluate the mathematical tractability of any model with dependent noise.

1991 Mathematics Subject Classification. Primary 60H15; Secondary 60G15. 
In the case of higher regularity $(H>1 / 2)$ the pathwise integrals led to the first results which established the existence of solutions to stochastic partial differential equations (SPDEs) (see Nualart and Răşcanu [18], or Garrido-Atienza et al. [7] for a different approach; infinite-dimensional equations have been treated with the same success as finite-dimensional ones, e.g. Grecksch and Anh [8], Maslowski and Nualart [13], Tindel et al. [19], Gubinelli et al. [9] and Garrido-Atienza et al. [6]).

On the other hand, retarded differential equations are an important area of applied mathematics due to physical reasons with non-instant transmission phenomena such as high velocity fields in wind tunnel experiments, or other memory processes (see, e.g., Hale and Verduyn Lunel [11] and Manitius [12]), or biological motivations like species growth or incubating time in disease models among many others (see Kuang [10] and Murray [15], for instance).

The asymptotic behavior of such models has meaningful interpretations like permanence, instability, and chaotic developments. In order to capture the stochasticity of such retarded systems, stochastic differential delay equations driven by the standard Brownian motion have been proposed and thoroughly investigated during the last decades. However, the literature about SDEs or SPDEs with delay driven by $\mathrm{fBm}$ is scarce. Yet observed retarded effect may be due just as much to longrange noise dependence as to deterministic delay. It is therefore important to find out how these two mechanisms interact.

In Ferrante and Rovira [4], the existence and uniqueness of solutions and the smoothness of the density for delayed SDEs driven by $\mathrm{fBm}$ is proved when $H>1 / 2$, but under strong hypotheses, using only techniques of the classical stochastic calculus, and preventing, for instance, the presence of a hereditary drift in the equations. In Neuenkirch et al. [16], using rough path theory, the authors prove existence and uniqueness of solutions to fractional equations with delays when $H>1 / 3$. More recently, Ferrante and Rovira [5] established the existence and uniqueness of solutions to delayed SDEs with fBm for $H>1 / 2$ and constant delay, by extending the results established in Nualart and Răşcanu [18], and the same sort of results have been shown recently for non-constant delay in Boufoussi and Hajji [2].

In this paper we consider the following stochastic semilinear delay evolution equation

$$
\left\{\begin{array}{l}
d X(t)=\left(A X(t)+f\left(t, X_{t}\right)\right) d t+g(t) d B_{Q}^{H}(t), \\
X(s)=\varphi(s),-r \leq s \leq 0, r \geq 0,
\end{array}\right.
$$

under suitable conditions on the operator $A$, the coefficient functions $f, g$, and the initial value $\varphi$. Here $B_{Q}^{H}(t)$ denotes an $\mathrm{fBm}$ with $H \in(1 / 2,1)$.

The purpose of this paper is to investigate existence and uniqueness of mild solutions to the fractional stochastic delay evolution equation (1.1) and to study its longtime behavior as well. We are clearly at the very beginning of the analysis of this realistic, important class of models. Beyond existence and uniqueness, one must investigate qualitative effects of solutions. We are also interested in analyzing if such equations generate random dynamical systems, and if so, whether there exist, for instance, random fixed points and random attractors (see Garrido-Atienza et al. [7] in the case of non-delay terms). Another interesting generalization is concerned with the case in which the delay also appears in the noisy term. These 
points will be the topic of forthcoming papers.

The contents of the paper are as follows. In Section 2 some necessary preliminaries on the stochastic integration with respect to $\mathrm{fBm}$ are established. Also a technical lemma which is crucial in our stability analysis is proved. In Section 3 the existence and uniqueness of mild solutions are proved. In Section 4 we prove that a mild solution, when it exists, is also a weak solution. The last section is devoted to the analysis of the asymptotic behavior of (1.1). Namely, we establish some sufficient conditions ensuring the exponential decay to zero in mean square of the mild solution of our delay model. Finally, we present two applications to the general theory: the cases of variable and distributed delay.

\section{Preliminaries}

In this section we introduce the fractional Brownian motion as well as the Wiener integral with respect to it. We also establish some important results which will be needed throughout the paper.

Let $(\Omega, \mathcal{F}, \mathbb{P})$ be a complete probability space.

Definition 1. Given $H \in(0,1)$, a continuous centered Gaussian process $\beta^{H}(t)$, $t \in \mathbb{R}$, with the covariance function

$$
R_{H}(t, s)=\mathbb{E}\left[\beta^{H}(t) \beta^{H}(s)\right]=\frac{1}{2}\left(|t|^{2 H}+|s|^{2 H}-|t-s|^{2 H}\right), \quad t, s \in \mathbb{R}
$$

is called a two-sided one-dimensional fractional Brownian motion ( $\mathrm{fBm}$ ), and $\mathrm{H}$ is the Hurst parameter.

Now we aim at introducing the Wiener integral with respect to the one-dimensional $\mathrm{fBm} \beta^{H}$. Let $T>0$ and denote by $\Lambda$ the linear space of $\mathbb{R}$-valued step functions on $[0, T]$, that is, $\phi \in \Lambda$ if

$$
\phi(t)=\sum_{i=1}^{n-1} x_{i} \chi_{\left[t_{i}, t_{i+1}\right)}(t),
$$

where $t \in[0, T], x_{i} \in \mathbb{R}$ and $0=t_{1}<t_{2}<\cdots<t_{n}=T$. For $\phi \in \Lambda$ we define its Wiener integral with respect to $\beta^{H}$ as

$$
\int_{0}^{T} \phi(s) d \beta^{H}(s)=\sum_{i=1}^{n-1} x_{i}\left(\beta^{H}\left(t_{i+1}\right)-\beta^{H}\left(t_{i}\right)\right) .
$$

Let $\mathcal{H}$ be the Hilbert space defined as the closure of $\Lambda$ with respect to the scalar product

$$
\left\langle\chi_{[0, t]}, \chi_{[0, s]}\right\rangle_{\mathcal{H}}=R_{H}(t, s) .
$$

Then the mapping

$$
\phi=\sum_{i=1}^{n-1} x_{i} \chi_{\left[t_{i}, t_{i+1}\right)} \mapsto \int_{0}^{T} \phi(s) d \beta^{H}(s)
$$

is an isometry between $\Lambda$ and the linear space $\operatorname{span}\left\{\beta^{H}, t \in[0, T]\right\}$, which can be extended to an isometry between $\mathcal{H}$ and the first Wiener chaos of the $\mathrm{fBm}$ $\overline{\operatorname{span}}^{L}(\Omega)\left\{\beta^{H}, t \in[0, T]\right\}$ (see Tindel et al. [19]). The image of an element $\varphi \in \mathcal{H}$ 
by this isometry is called the Wiener integral of $\varphi$ with respect to $\beta^{H}$. Our next goal is to give an explicit expression of this integral. To this end, consider the kernel

$$
K_{H}(t, s)=c_{H} s^{1 / 2-H} \int_{s}^{t}(u-s)^{H-3 / 2} u^{H-1 / 2} d u
$$

where $c_{H}=\left(\frac{H(2 H-1)}{B\left(2-2 H, H-\frac{1}{2}\right)}\right)^{1 / 2}$, with $B$ denoting the Beta function, and $t>s$. It is not difficult to see that

$$
\frac{\partial K_{H}}{\partial t}(t, s)=c_{H}\left(\frac{t}{s}\right)^{H-1 / 2}(t-s)^{H-3 / 2} .
$$

Consider the linear operator $K_{H}^{*}: \Lambda \mapsto L^{2}([0, T])$ given by

$$
\left(K_{H}^{*} \phi\right)(s)=\int_{s}^{t} \phi(t) \frac{\partial K_{H}}{\partial t}(t, s) d t
$$

Then

$$
\left(K_{H}^{*} \chi_{[0, t]}\right)(s)=K_{H}(t, s) \chi_{[0, t]}(s)
$$

and $K_{H}^{*}$ is an isometry between $\Lambda$ and $L^{2}([0, T])$ that can be extended to $\mathcal{H}$ (see Alos et al. [1]).

Considering $W=\{W(t), t \in[0, T]\}$ defined by

$$
W(t)=\beta^{H}\left(\left(K_{H}^{*}\right)^{-1} \chi_{[0, t]}\right),
$$

it turns out that $W$ is a Wiener process and $\beta^{H}$ has the following Wiener integral representation:

$$
\beta^{H}(t)=\int_{0}^{t} K_{H}(t, s) d W(s) .
$$

In addition, for any $\phi \in \mathcal{H}$,

$$
\int_{0}^{T} \phi(s) d \beta^{H}(s)=\int_{0}^{T}\left(K_{H}^{*} \phi\right)(t) d W(t)
$$

if and only if $K_{H}^{*} \phi \in L^{2}([0, T])$.

Also denoting $L_{\mathcal{H}}^{2}([0, T])=\left\{\phi \in \mathcal{H}, K_{H}^{*} \phi \in L^{2}([0, T])\right\}$, since $H>1 / 2$, we have

$$
L^{1 / H}([0, T]) \subset L_{\mathcal{H}}^{2}([0, T]),
$$

see Mishura [14]. Moreover, the following useful result holds:

Lemma 1. (Nualart $[17])$ For $\phi \in L^{1 / H}([0, T])$,

$$
H(2 H-1) \int_{0}^{T} \int_{0}^{T}|\phi(r)\|\phi(u)\| r-u|^{2 H-2} d r d u \leq c_{H}\|\phi\|_{L^{1 / H}([0, T])}^{2} .
$$

Next we are interested in considering an fBm with values in a Hilbert space and giving the definition of the corresponding stochastic integral.

Let $\left(U,|\cdot|_{U},(\cdot, \cdot)_{U}\right)$ and $\left(K,|\cdot|_{K},(\cdot, \cdot)_{K}\right)$ be separable Hilbert spaces. Let $L(K, U)$ denote the space of all bounded linear operators from $K$ to $U$. Let $Q \in L(K, K)$ be a nonnegative self-adjoint operator. Denote by $L_{Q}^{0}(K, U)$ the space of all $\xi \in L(K, U)$ such that $\xi Q^{\frac{1}{2}}$ is a Hilbert-Schmidt operator. The norm is given by

$$
|\xi|_{L_{Q}^{0}(K, U)}^{2}=\left|\xi Q^{\frac{1}{2}}\right|_{H S}^{2}=\operatorname{tr}\left(\xi Q \xi^{*}\right) .
$$


Then $\xi$ is called a $Q$-Hilbert-Schmidt operator from $K$ to $U$.

Let $\left\{\beta_{n}^{H}(t)\right\}_{n \in \mathbb{N}}$ be a sequence of two-sided one-dimensional standard fractional Brownian motions mutually independent on $(\Omega, \mathcal{F}, \mathbb{P})$. When one considers the following series

$$
\sum_{n=1}^{\infty} \beta_{n}^{H}(t) e_{n}, t \geq 0
$$

where $\left\{e_{n}\right\}_{n \in \mathbb{N}}$ is a complete orthonormal basis in $K$, this series does not necessarily converge in the space $K$. Thus we consider a $K$-valued stochastic process $B_{Q}^{H}(t)$ given formally by the following series:

$$
B_{Q}^{H}(t)=\sum_{n=1}^{\infty} \beta_{n}^{H}(t) Q^{\frac{1}{2}} e_{n}, t \geq 0 .
$$

If $Q$ is a nonnegative self-adjoint trace class operator, then this series converges in the space $K$, that is, it holds that $B_{Q}^{H}(t) \in L^{2}(\Omega, K)$. Then, we say that the above $B_{Q}^{H}(t)$ is a $K$-valued $Q$-cylindrical fractional Brownian motion with covariance operator $Q$. For example, if $\left\{\sigma_{n}\right\}_{n \in \mathbb{N}}$ is a bounded sequence of non-negative real numbers such that $Q e_{n}=\sigma_{n} e_{n}$, assuming that $Q$ is a nuclear operator in $K$ (that is, $\left.\sum_{n=1}^{\infty} \sigma_{n}<\infty\right)$, then the stochastic process

$$
B_{Q}^{H}(t)=\sum_{n=1}^{\infty} \beta_{n}^{H}(t) Q^{\frac{1}{2}} e_{n}=\sum_{n=1}^{\infty} \sqrt{\sigma_{n}} \beta_{n}^{H}(t) e_{n}, t \geq 0,
$$

is well defined as a $K$-valued $Q$-cylindrical fractional Brownian motion.

Let $\varphi:[0, T] \rightarrow L_{Q}^{0}(K, U)$ such that

$$
\sum_{n=1}^{\infty}\left\|K_{H}^{*}\left(\varphi Q^{1 / 2} e_{n}\right)\right\|_{L^{2}([0, T] ; U)}<\infty .
$$

Definition 2. Let $\varphi:[0, T] \rightarrow L_{Q}^{0}(K, U)$ satisfy (2.2). Then, its stochastic integral with respect to the $f B m B_{Q}^{H}$ is defined, for $t \geq 0$, as follows

$$
\int_{0}^{t} \varphi(s) d B_{Q}^{H}(s):=\sum_{n=1}^{\infty} \int_{0}^{t} \varphi(s) Q^{1 / 2} e_{n} d \beta_{n}^{H}=\sum_{n=1}^{\infty} \int_{0}^{t}\left(K_{H}^{*}\left(\varphi Q^{1 / 2} e_{n}\right)\right)(s) d W(s) .
$$

Notice that if

$$
\sum_{n=1}^{\infty}\left\|\varphi Q^{1 / 2} e_{n}\right\|_{L^{1 / H}([0, T] ; U)}<\infty,
$$

then in particular (2.2) holds, which follows immediately from (2.1).

The following lemma is obtained as a simple application of Lemma 1.

Lemma 2. For any $\varphi:[0, T] \mapsto L_{Q}^{0}(K, U)$ such that (2.3) holds, and for any $\alpha, \beta \in[0, T]$ with $\alpha>\beta$,

$$
\mathbb{E}\left|\int_{\beta}^{\alpha} \varphi(s) d B_{Q}^{H}(s)\right|_{U}^{2} \leq c H(2 H-1)(\alpha-\beta)^{2 H-1} \sum_{n=1}^{\infty} \int_{\beta}^{\alpha}\left|\varphi(s) Q^{1 / 2} e_{n}\right|_{U}^{2} d s,
$$


where $c=c(H)$. If, in addition,

$$
\sum_{n=1}^{\infty}\left|\varphi(t) Q^{1 / 2} e_{n}\right|_{U} \text { is uniformly convergent for } t \in[0, T]
$$

then

$$
\mathbb{E}\left|\int_{\beta}^{\alpha} \varphi(s) d B_{Q}^{H}(s)\right|_{U}^{2} \leq c H(2 H-1)(\alpha-\beta)^{2 H-1} \int_{\beta}^{\alpha}|\varphi(s)|_{L_{Q}^{0}(K, U)}^{2} d s .
$$

Proof. Let $\left\{e_{n}\right\}_{n \in \mathbb{N}}$ be the complete orthonormal basis of $K$ introduced above. Applying Lemma 1 we obtain

$$
\begin{aligned}
& \mathbb{E}\left|\int_{\beta}^{\alpha} \varphi(s) d B_{Q}^{H}(s)\right|_{U}^{2} \\
= & \mathbb{E}\left|\sum_{n=1}^{\infty} \int_{\beta}^{\alpha} \varphi(s) Q^{\frac{1}{2}} e_{n} d \beta_{n}^{H}(s)\right|_{U}^{2} \\
= & \sum_{n=1}^{\infty} \mathbb{E}\left|\int_{\beta}^{\alpha} \varphi(s) Q^{\frac{1}{2}} e_{n} d \beta_{n}^{H}(s)\right|_{U}^{2} \\
= & \sum_{n=1}^{\infty} H(2 H-1) \int_{\beta}^{\alpha} \int_{\beta}^{\alpha}\left|\varphi(t) Q^{\frac{1}{2}} e_{n}\right|_{U}\left|\varphi(s) Q^{\frac{1}{2}} e_{n}\right|_{U}|t-s|^{2 H-2} d t d s \\
\leq & c H(2 H-1) \sum_{n=1}^{\infty}\left(\int_{\beta}^{\alpha}\left|\varphi(s) Q^{1 / 2} e_{n}\right|_{U}^{1 / H} d s\right)^{2 H} \\
\leq & c H(2 H-1)(\alpha-\beta)^{2 H-1} \sum_{n=1}^{\infty} \int_{\beta}^{\alpha}\left|\varphi(s) Q^{1 / 2} e_{n}\right|_{U}^{2} d s .
\end{aligned}
$$

The second assertion is an immediate consequence of the Weierstrass M-test.

Remark 1. If $\left\{\sigma_{n}\right\}_{n \in \mathbb{N}}$ is a bounded sequence of non-negative real numbers such that the nuclear operator $Q$ satisfies $Q e_{n}=\sigma_{n} e_{n}$, assuming that there exists a positive constant $k_{\varphi}$ such that

$$
|\varphi(t)|_{L_{Q}^{2}(K, U)} \leq k_{\varphi}, \quad \text { uniformly in }[0, T],
$$

then (2.4) holds automatically.

\section{EXISTENCE AND UNIQUENESS OF MILD SOLUTION}

Consider $(\Omega, \mathcal{F}, \mathbb{P})$, the complete probability space which was introduced in Section 2. Denote $\mathcal{F}_{t}=\mathcal{F}_{0}$, for all $t \leq 0$.

We denote by $C\left(a, b ; L^{2}(\Omega ; U)\right)=C\left(a, b ; L^{2}(\Omega, \mathcal{F}, \mathbb{P} ; U)\right)$ the Banach space of all continuous functions from $[a, b]$ into $L^{2}(\Omega ; U)$ equipped with the sup norm.

Let us also consider two fixed real numbers $r \geq 0$ and $T>0$. If $x \in C\left(-r, T ; L^{2}(\Omega ; U)\right)$ for each $t \in[0, T]$ we denote by $x_{t} \in C\left(-r, 0 ; L^{2}(\Omega ; U)\right)$ the function defined by $x_{t}(s)=x(t+s)$, for $s \in[-r, 0]$.

In this section we consider the existence and uniqueness of mild solutions to the following stochastic evolution equation with delays:

$$
\left\{\begin{array}{l}
d X(t)=\left(A X(t)+f\left(t, X_{t}\right)\right) d t+g(t) d B_{Q}^{H}(t), \quad t \in[0, T], \\
X(t)=\varphi(t), \quad t \in[-r, 0],
\end{array}\right.
$$


where $B_{Q}^{H}(t)$ is the fractional Brownian motion which was introduced in the previous section, the initial data $\varphi \in C\left(-r, 0 ; L^{2}(\Omega ; U)\right)$ and $A: \operatorname{Dom}(A) \subset U \rightarrow U$ is the infinitesimal generator of a strongly continuous semigroup $S(\cdot)$ on $U$, that is, for $t \geq 0$, it holds

$$
|S(t)|_{U} \leq M e^{\rho t}, \quad M \geq 1, \rho \in \mathbb{R} .
$$

$f:[0, T] \times C(-r, 0 ; U) \rightarrow U$ is a family of non-linear operators defined for almost every $t$ (a.e. $t)$ which satisfies

( $f$.1) The mapping $t \in(0, T) \rightarrow f(t, \xi) \in U$ is Lebesgue measurable, for a.e. $t$ and for all $\xi \in C(-r, 0 ; U)$.

$(f .2)$ There exists a constant $C_{f}>0$ such that for any $x, y \in C(-r, T ; U)$ and $t \in[0, T]$,

$$
\int_{0}^{t}\left|f\left(s, x_{s}\right)-f\left(s, y_{s}\right)\right|_{U}^{2} d s \leq C_{f} \int_{-r}^{t}|x(s)-y(s)|_{U}^{2} d s .
$$

$$
\int_{0}^{T}|f(s, 0)|_{U}^{2} d s<\infty
$$

Moreover, for $g:[0, T] \rightarrow L_{Q}^{0}(K, U)$ we assume the following conditions: for the complete orthonormal basis $\left\{e_{n}\right\}_{n \in \mathbb{N}}$ in $K$, we have

$$
\begin{aligned}
& \text { (g.1) } \sum_{n=1}^{\infty}\left\|g Q^{1 / 2} e_{n}\right\|_{L^{2}([0, T] ; U)}<\infty . \\
& \text { (g.2) } \sum_{n=1}^{\infty}\left|g(t) Q^{1 / 2} e_{n}\right|_{U} \text { is uniformly convergent for } t \in[0, T] .
\end{aligned}
$$

Definition 3. A $U$-valued process $X(t)$ is called a mild solution of (3.1) if $X \in$ $C\left(-r, T ; L^{2}(\Omega ; U)\right), X(t)=\varphi(t)$ for $t \in[-r, 0]$, and, for $t \in[0, T]$, satisfies

$$
X(t)=S(t) \varphi(0)+\int_{0}^{t} S(t-s) f\left(s, X_{s}\right) d s+\int_{0}^{t} S(t-s) g(s) d B_{Q}^{H}(s) \quad \mathbb{P}-a . s .
$$

Notice that, thanks to $(g .1)$ and the fact that $H \in(1 / 2,1),(2.3)$ holds, which implies that the stochastic integral in (3.2) is well-defined since $S(\cdot)$ is a strongly continuous semigroup. Moreover, $(g .1)$ together with $(g .2)$ immediately imply that, for every $t \in[0, T]$,

$$
\int_{0}^{t}|g(s)|_{L_{Q}^{0}(K, U)}^{2} d s<\infty
$$

Theorem 1. Under the assumptions on $A$ and conditions $(f .1)-(f .3)$ and $(g .1)$ $(g .2)$, for every $\varphi \in C\left(-r, 0 ; L^{2}(\Omega ; U)\right)$ there exists a unique mild solution $X$ to (3.1).

Proof. We can a assume that $\rho>0$, otherwise we can take $\rho_{0}>0$ such that, for $t \geq 0,|S(t)| \leq M e^{\rho_{0} t}$. 
We start the proof by checking the uniqueness of solutions. Assume that $X, Y \in$ $C\left(-r, T ; L^{2}(\Omega ; U)\right)$ are two mild solutions of (3.1). Then,

$$
\begin{aligned}
\mathbb{E}|X(t)-Y(t)|_{U}^{2} & \leq t \mathbb{E} \int_{0}^{t}\left|S(t-s)\left(f\left(s, X_{s}\right)-f\left(s, Y_{s}\right)\right)\right|_{U}^{2} d s \\
& \leq t M^{2} e^{2 \rho t} \mathbb{E} \int_{0}^{t}\left|f\left(s, X_{s}\right)-f\left(s, Y_{s}\right)\right|_{U}^{2} d s \\
& \leq t M^{2} e^{2 \rho t} C_{f} \int_{0}^{t} \mathbb{E}|X(s)-Y(s)|_{U}^{2} d s \\
& \leq t M^{2} e^{2 \rho t} C_{f} \int_{0}^{t} \sup _{0 \leq \tau \leq s} \mathbb{E}|X(\tau)-Y(\tau)|_{U}^{2} d s
\end{aligned}
$$

and therefore, since $X=Y$ over the interval $[-r, 0]$, by taking supremum in the above inequality,

$$
\sup _{0 \leq \theta \leq t} \mathbb{E}|X(\theta)-Y(\theta)|_{U}^{2} \leq T M^{2} e^{2 \rho T} C_{f} \int_{0}^{t} \sup _{0 \leq \tau \leq s} \mathbb{E}|X(\tau)-Y(\tau)|_{U}^{2} d s .
$$

The Gronwall Lemma implies now the uniqueness result.

Now we prove the existence of solutions to problem (3.1).

First of all, we check that the well-defined stochastic integral possesses the required regularity. To do that, let us consider $\sigma>0$ small enough. We have

$$
\begin{aligned}
& \mathbb{E}\left|\int_{0}^{t+\sigma} S(t+\sigma-s) g(s) d B_{Q}^{H}(s)-\int_{0}^{t} S(t-s) g(s) d B_{Q}^{H}(s)\right|^{2} \\
\leq & 2 \mathbb{E}\left|\int_{0}^{t}(S(t+\sigma-s)-S(t-s)) g(s) d B_{Q}^{H}(s)\right|^{2}+2 \mathbb{E}\left|\int_{t}^{t+\sigma} S(t-s) g(s) d B_{Q}^{H}(s)\right|^{2} \\
:= & J_{1}+J_{2} .
\end{aligned}
$$

Firstly, applying inequality (2.5) to $J_{1}$,

$$
\begin{aligned}
J_{1} & \leq 2 c H(2 H-1) t^{2 H-1} \int_{0}^{t}|S(t-s)(S(\sigma)-\mathrm{Id}) g(s)|_{L_{Q}^{0}(K, U)}^{2} d s \\
& \leq 2 c H(2 H-1) t^{2 H-1} M^{2} e^{2 \rho t} \int_{0}^{t}|(S(\sigma)-\mathrm{Id}) g(s)|_{L_{Q}^{0}(K, U)}^{2} d s \rightarrow 0
\end{aligned}
$$

when $\sigma \rightarrow 0$ thanks to the Lebesgue majorant Theorem, since, for every $s$ fixed,

$$
\begin{aligned}
S(\sigma) g(s) & \rightarrow g(s), \\
|S(\sigma) g(s)|_{L_{Q}^{0}(K, U)} & \leq M e^{\rho \sigma}|g(s)|_{L_{Q}^{0}(K, U)} .
\end{aligned}
$$

Applying now (2.5) to $J_{2}$ we obtain

$$
J_{2} \leq 2 c H(2 H-1) \sigma^{2 H-1} M^{2} e^{2 \rho \sigma} \int_{t}^{t+\sigma}|g(s)|_{L_{Q}^{0}(K, U)}^{2} d s \rightarrow 0
$$

when $\sigma \rightarrow 0$. Therefore the stochastic integral belongs to the space $C\left(-r, T ; L^{2}(\Omega ; U)\right)$.

We denote $X^{0}=0$ and define by recurrence a sequence $\left\{X^{n}\right\}_{n \in \mathbb{N}}$ of processes as 


$$
\left\{\begin{aligned}
X^{n}(t) & =S(t) \varphi(0)+\int_{0}^{t} S(t-s) f\left(s, X_{s}^{n-1}\right) d s \\
& +\int_{0}^{t} S(t-s) g(s) d B_{Q}^{H}(s), t \in[0, T], \\
X^{n}(t) & =\varphi(t), t \in[-r, 0] .
\end{aligned}\right.
$$

The sequence (3.3) is well-defined, since $X^{0}=0 \in C\left(-r, T ; L^{2}(\Omega ; U)\right)$ and, given $X^{n-1} \in C\left(-r, T ; L^{2}(\Omega ; U)\right)$, let us check that $X^{n} \in C\left(-r, T ; L^{2}(\Omega ; U)\right)$ as well.

In order to prove the previous assertion, let us consider again $\sigma>0$ sufficiently small. Then

$$
\begin{aligned}
\left|X^{n}(t+\sigma)-X^{n}(t)\right|_{U}^{2} & \leq 2\left|\int_{0}^{t}(S(t+\sigma-s)-S(t-s)) f\left(s, X_{s}^{n-1}\right) d s\right|_{U}^{2} \\
& +2\left|\int_{t}^{t+\sigma} S(t+\sigma-s) f\left(s, X_{s}^{n-1}\right) d s\right|_{U}^{2} \\
& :=I_{1}+I_{2} .
\end{aligned}
$$

On the one hand,

$$
\mathbb{E} I_{1} \leq 2 M^{2} t e^{2 \rho t} \mathbb{E} \int_{0}^{t}\left|(S(\sigma)-\mathrm{Id}) f\left(s, X_{s}^{n-1}\right)\right|_{U}^{2} d s \rightarrow 0
$$

when $\sigma \rightarrow 0$ thanks again to the Lebesgue majorant Theorem, since, for each $s$ fixed,

$$
\begin{aligned}
S(\sigma) f\left(s, X_{s}^{n-1}\right) & \rightarrow f\left(s, X_{s}^{n-1}\right), \\
\left|S(\sigma) f\left(s, X_{s}^{n-1}\right)\right|_{U} & \leq M e^{\rho \sigma}\left|f\left(s, X_{s}^{n-1}\right)\right|_{U},
\end{aligned}
$$

and

$$
\mathbb{E} \int_{0}^{t}\left|f\left(s, X_{s}^{n-1}\right)\right|_{U} d s \leq \mathbb{E} \int_{-r}^{t}\left|X^{n-1}(s)\right|_{U} d s+\mathbb{E} \int_{0}^{t}|f(s, 0)|_{U} d s<\infty,
$$

due to conditions $(f .2)$ and $(f .3)$ and the fact that $X^{n-1} \in C\left(-r, T ; L^{2}(\Omega ; U)\right)$. On the other hand,

$$
\begin{aligned}
I_{2} & \leq 2 \sigma M^{2} e^{2 \rho \sigma} \int_{t}^{t+\sigma}\left|f\left(s, X_{s}^{n-1}\right)-f(s, 0)\right|_{U}^{2} d s+2 \sigma M^{2} e^{2 \rho \sigma} \int_{t}^{t+\sigma}|f(s, 0)|_{U}^{2} d s \\
& \leq 2 \sigma M^{2} e^{2 \rho \sigma} C_{f} \int_{-r}^{t+\sigma}\left|X^{n-1}(s)\right|_{U}^{2} d s+2 \sigma M^{2} e^{2 \rho \sigma} \int_{t}^{t+\sigma}|f(s, 0)|_{U}^{2} d s,
\end{aligned}
$$

so that

$$
\mathbb{E} I_{2} \leq 2 \sigma M^{2} e^{2 \rho \sigma} C_{f} \int_{-r}^{t+\sigma} \mathbb{E}\left|X^{n-1}(s)\right|_{U}^{2} d s+2 \sigma M^{2} e^{2 \rho \sigma} \int_{t}^{t+\sigma}|f(s, 0)|_{U}^{2} d s \rightarrow 0
$$

when $\sigma \rightarrow 0$.

We want to show now that $\left\{X^{n}\right\}_{n \in \mathbb{N}}$ is a Cauchy sequence in $C\left(-r, T ; L^{2}(\Omega ; U)\right)$.

Firstly, for $t \in[0, T]$ and $n \in \mathbb{N}$, since $X^{n}=X^{n-1}$ on $[-r, 0]$, it holds

$$
\left|X^{n+1}(t)-X^{n}(t)\right|_{U}^{2} \leq t M^{2} e^{2 \rho t} C_{f} \int_{0}^{t}\left|X^{n}(s)-X^{n-1}(s)\right|_{U}^{2} d s
$$


and this, implies

$$
\mathbb{E}\left|X^{n+1}(t)-X^{n}(t)\right|_{U}^{2} \leq t M^{2} e^{2 \rho t} C_{f} \int_{0}^{t} \sup _{0 \leq \tau \leq s} \mathbb{E}\left|X^{n}(\tau)-X^{n-1}(\tau)\right|_{U}^{2} d s
$$

Defining

$$
\mathcal{X}^{n}(t)=\sup _{0 \leq \theta \leq t} \mathbb{E}\left|X^{n+1}(\theta)-X^{n}(\theta)\right|_{U}^{2}
$$

we have

$$
\mathcal{X}^{n}(t) \leq k \int_{0}^{t} \mathcal{X}^{n-1}(s) d s, \forall n \geq 2
$$

for $k=T M^{2} e^{2 \rho T} C_{f}$. Consequently, by iteration we obtain

$$
\mathcal{X}^{n}(t) \leq \frac{k^{n-1} T^{n-1}}{(n-1) !} \mathcal{X}^{1}(T), \forall n \geq 2, \forall t \in[0, T]
$$

Since $X^{n+1}(t)=X^{n}(t), \forall t \in[-r, 0]$, the last estimate implies that $\left\{X^{n}\right\}_{n \in \mathbb{N}}$ is a Cauchy sequence in $C\left(-r, T ; L^{2}(\Omega ; U)\right)$.

Finally, we check that the limit $X$ of the sequence $\left\{X^{n}\right\}_{n \in \mathbb{N}}$ is a solution of (3.1). But, this is straightforward taking into account that $X^{n}$ is defined by (3.3) and that $f$ satisfies $(f .2)$, so that, in particular, when $n \rightarrow \infty$,

$\mathbb{E}\left|\int_{0}^{t} S(t-s)\left(f\left(s, X_{s}^{n-1}\right)-f\left(s, X_{s}\right)\right) d s\right|_{U}^{2} \leq t M^{2} e^{2 \rho t} C_{f} \int_{0}^{t} \mathbb{E}\left|X^{n-1}(s)-X(s)\right|_{U}^{2} d s \rightarrow 0$,

and therefore $X$ is the unique (mild) solution of (3.1).

\section{EXistence OF WEAK SOLUtions}

In this section we prove that the mild solution to system (3.1) is also a weak solution. First of all we recall the definition of weak solution according to Da Prato and Zabczyk [3].

To shorten the notation, we will use $<\cdot, \cdot>$ instead of $(\cdot, \cdot)_{U}$ below.

Definition 4. An $U$-valued process $X(t), t \in[-r, T]$, is called a weak solution of (3.1) if $X(t)=\varphi(t)$, for $t \in[-r, 0]$, and for all $\zeta \in D\left(A^{*}\right)$ and all $t \in[0, T]$

$\langle X(t), \zeta\rangle=\langle\varphi(0), \zeta\rangle+\int_{0}^{t}\left(\left\langle X(\tau), A^{*} \zeta\right\rangle+\left\langle f\left(\tau, X_{\tau}\right), \zeta\right\rangle\right) d \tau+\int_{0}^{t}\langle g(\tau), \zeta\rangle d B_{Q}^{H}(\tau) \quad \mathbb{P}-$ a.s.

Theorem 2. Under the assumptions of Theorem 1, the mild solution of (3.1) is also a weak solution. 
Proof. Since $X(t)$ is a mild solution, for each $\zeta \in D\left(A^{*}\right)$ it follows that

$$
\begin{aligned}
\mathbb{E} & {\left[\mid \int_{0}^{t}\left\langle X(s), A^{*} \zeta\right\rangle d s-\int_{0}^{t}\left\langle S(s) \varphi(0), A^{*} \zeta\right\rangle d s\right.} \\
& -\int_{0}^{t} \int_{0}^{s}\left\langle S(s-\tau) f\left(\tau, X_{\tau}\right), A^{*} \zeta\right\rangle d \tau d s \\
& \left.-\int_{0}^{t} \int_{0}^{s}\left\langle S(s-\tau) g(\tau), A^{*} \zeta\right\rangle d B_{Q}^{H}(\tau) d s \mid\right] \\
& \leq \int_{0}^{t} \mathbb{E}\left[\mid\left\langle X(s), A^{*} \zeta\right\rangle-\left\langle S(s) \varphi(0), A^{*} \zeta\right\rangle-\int_{0}^{s}\left\langle S(s-\tau) f\left(\tau, X_{\tau}\right), A^{*} \zeta\right\rangle d \tau\right. \\
& \left.-\int_{0}^{s}\left\langle S(s-\tau) g(\tau), A^{*} \zeta\right\rangle d B_{Q}^{H}(\tau) \mid\right] d s \\
& =\int_{0}^{t} \mathbb{E}\left[\mid\left\langle X(s)-S(s) \varphi(0)-\int_{0}^{s} S(s-\tau) f\left(\tau, X_{\tau}\right) d \tau\right.\right. \\
& \left.\left.-\int_{0}^{s} S(s-\tau) g(\tau) d B_{Q}^{H}(\tau), A^{*} \zeta\right\rangle \mid\right] d s \\
& =0 .
\end{aligned}
$$

Thus, for a.e. $\omega \in \Omega$, it holds

$$
\begin{aligned}
\int_{0}^{t}\left\langle X(s), A^{*} \zeta\right\rangle d s & =\int_{0}^{t}\left\langle S(s) \varphi(0), A^{*} \zeta\right\rangle d s+\int_{0}^{t} \int_{0}^{s}\left\langle S(s-\tau) f\left(\tau, X_{\tau}\right), A^{*} \zeta\right\rangle d \tau d s \\
& +\int_{0}^{t} \int_{0}^{s}\left\langle S(s-\tau) g(\tau), A^{*} \zeta\right\rangle d B_{Q}^{H}(\tau) d s .
\end{aligned}
$$

Now we use that, for $\zeta \in D\left(A^{*}\right), \frac{d}{d t} S^{*}(t) \zeta=S^{*}(t) A^{*} \zeta$. We firstly obtain

$$
\begin{aligned}
\int_{0}^{t}\left\langle S(s) \varphi(0), A^{*} \zeta\right\rangle d s & =\int_{0}^{t}\left\langle\varphi(0), S^{*}(s) A^{*} \zeta\right\rangle d s \\
& =\langle S(t) \varphi(0)-\varphi(0), \zeta\rangle
\end{aligned}
$$

and, in addition, using Fubini's Theorem,

$$
\begin{aligned}
& \int_{0}^{t} \int_{0}^{s}\left\langle S(s-\tau) f\left(\tau, X_{\tau}\right), A^{*} \zeta\right\rangle d \tau d s \\
= & \int_{0}^{t} \int_{\tau}^{t}\left\langle 1_{(0, s]}(\tau) f\left(\tau, X_{\tau}\right), S^{*}(s-\tau) A^{*} \zeta\right\rangle d s d \tau \\
= & \int_{0}^{t}\left\langle S(t-\tau) f\left(\tau, X_{\tau}\right)-f\left(\tau, X_{\tau}\right), \zeta\right\rangle d \tau .
\end{aligned}
$$


Finally,

$$
\begin{aligned}
& \int_{0}^{t} \int_{0}^{s}\left\langle S(s-\tau) g(\tau), A^{*} \zeta\right\rangle d B_{Q}^{H}(\tau) d s \\
= & \int_{0}^{t} \int_{\tau}^{t}\left\langle 1_{(0, s]}(\tau) g(\tau), S^{*}(s-\tau) A^{*} \zeta\right\rangle d s d B_{Q}^{H}(\tau) \\
= & \int_{0}^{t}\left\langle g(\tau), S^{*}(t-\tau) \zeta-\zeta\right\rangle d B_{Q}^{H}(\tau) \\
= & \int_{0}^{t}\langle S(t-\tau) g(\tau), \zeta\rangle d B_{Q}^{H}(\tau)-\int_{0}^{t}\langle g(\tau), \zeta\rangle d B_{Q}^{H}(\tau) .
\end{aligned}
$$

Therefore by (4.1) for a.e. $\omega \in \Omega$, it follows

$$
\begin{aligned}
& \int_{0}^{t}\langle A X(s), \zeta\rangle d s=\int_{0}^{t}\left\langle X(s), A^{*} \zeta\right\rangle d s \\
= & \langle S(t) \varphi(0)-\varphi(0), \zeta\rangle+\int_{0}^{t}\left\langle S(t-\tau) f\left(\tau, X_{\tau}\right)-f\left(\tau, X_{\tau}\right), \zeta\right\rangle d \tau \\
& +\int_{0}^{t}\langle S(t-\tau) g(\tau), \zeta\rangle d B_{Q}^{H}(\tau)-\int_{0}^{t}\langle g(\tau), \zeta\rangle d B_{Q}^{H}(\tau) \\
= & \langle X(t)-\varphi(0), \zeta\rangle-\int_{0}^{t}\left\langle f\left(\tau, X_{\tau}\right) d \tau, \zeta\right\rangle-\int_{0}^{t}\left\langle g(\tau) d B_{Q}^{H}(\tau), \zeta\right\rangle .
\end{aligned}
$$

Consequently, it follows that almost surely

$$
\langle X(t), \zeta\rangle=\langle\varphi(0), \zeta\rangle+\int_{0}^{t}\left(\left\langle X(\tau), A^{*} \zeta\right\rangle+\left\langle f\left(\tau, X_{\tau}\right), \zeta\right\rangle\right) d \tau+\int_{0}^{t}\langle g(\tau), \zeta\rangle d B_{Q}^{H}(\tau),
$$

which means that $X(t)$ is the weak solution to $(3.1)$.

\section{EXPONEnTIAL DECAY OF SOLUTIONS IN MEAN SQUARE}

As in this section we are interested in the exponential decay to zero in mean square of the mild solutions to (3.1), we shall therefore assume that for each $T>0$ and for each $\varphi \in C\left(-r, 0 ; L^{2}(\Omega ; U)\right)$, problem (3.1) possesses a unique mild solution according to the Definition 3.

We first need to state the following conditions:

Condition 1. The operator $A$ is a closed linear operator generating a strongly continuous semigroup $S(t), t \geq 0$, on the separable Hilbert space $U$ and satisfies

$$
|S(t)|_{U} \leq M e^{-\lambda t}, \forall t \geq 0, \text { where } M \geq 1, \lambda>0 .
$$

Condition 2. There exists a constant $C_{f} \geq 0$, such that, for any $x, y \in C(-r, T ; U)$, and for all $t \geq 0$

$$
\int_{0}^{t} e^{m s}\left|f\left(s, x_{s}\right)-f\left(s, y_{s}\right)\right|_{U}^{2} d s \leq C_{f} \int_{-r}^{t} e^{m s}|x(s)-y(s)|_{U}^{2} d s, \quad \text { for all } 0 \leq m \leq \lambda
$$

and

$$
\int_{0}^{\infty} e^{\lambda s}|f(s, 0)|_{U}^{2} d s<\infty
$$


Condition 3. In addition to assumptions (g.1) and (g.2), assume

$$
\int_{0}^{\infty} e^{\lambda s}|g(s)|_{L_{Q}^{0}(K, U)}^{2} d s<\infty .
$$

The following theorem shows the exponential decay to zero in mean square, with an explicit exponential decay rate $\gamma$.

Theorem 3. In addition to Conditions 1-3, assume that the mild solution $X(t)$ of system (3.1) corresponding to the initial function $\varphi \in C\left(-r, 0 ; L^{2}(\Omega ; U)\right)$, exists for all $t \geq-r$, and that

$$
\lambda^{2}>6 C_{f} M^{2} .
$$

Then, there exists a constant $\gamma>0$ such that

$$
\limsup _{t \rightarrow \infty}\left(\frac{1}{t}\right) \log \mathbb{E}|X(t)|_{U}^{2} \leq-\gamma .
$$

In other words, every mild solution exponentially decays to zero in mean square.

Proof. Thanks to the fact that $\lambda^{2}>6 C M^{2}$, we can choose $\theta>0$ such that $\gamma=$ $\lambda-\theta-6 M^{2} C_{f} \lambda^{-1}>0$. Then, for this $\gamma$ we have

$$
\begin{aligned}
& \mathbb{E}|X(t)|_{U}^{2} \leq 3 \mathbb{E}|S(t) \varphi(0)|_{U}^{2}+3 \mathbb{E}\left|\int_{0}^{t} S(t-s) f\left(s, X_{s}\right) d s\right|_{U}^{2} \\
& +3 \mathbb{E}\left|\int_{0}^{t} S(t-s) g(s) d B_{Q}^{H}(s)\right|_{U}^{2} .
\end{aligned}
$$

Therefore, by Condition 1 and Lemma 2

$$
\begin{aligned}
& \mathbb{E}|X(t)|_{U}^{2} \leq 3 \mathbb{E}|S(t) \varphi(0)|_{U}^{2} \\
& +3 M^{2} \int_{0}^{t} \mathrm{e}^{-\lambda(t-s)} d s \int_{0}^{t} \mathrm{e}^{-\lambda(t-s)} \mathbb{E}\left|f\left(s, X_{s}\right)\right|_{U}^{2} d s \\
& +3 c H(2 H-1) M^{2} t^{2 H-1} \int_{0}^{t} \mathrm{e}^{-2 \lambda(t-s)}|g(s)|_{L_{Q}^{0}(K, U)}^{2} d s \\
& \leq 3 M^{2} \mathrm{e}^{-2 \lambda t} \mathbb{E}|\varphi(0)|_{U}^{2}+\frac{3}{\lambda} M^{2} \int_{0}^{t} \mathrm{e}^{-\lambda(t-s)} \mathbb{E}\left|f\left(s, X_{s}\right)\right|_{U}^{2} d s \\
& +3 c H(2 H-1) M^{2} t^{2 H-1} \int_{0}^{t} \mathrm{e}^{-2 \lambda(t-s)}|g(s)|_{L_{Q}^{0}(K, U)}^{2} d s,
\end{aligned}
$$

and, consequently,

$$
\begin{aligned}
\mathrm{e}^{\lambda t} \mathbb{E}|X(t)|_{U}^{2} \leq & 3 M^{2} \mathbb{E}|\varphi(0)|_{U}^{2}+\frac{3}{\lambda} M^{2} \int_{0}^{t} \mathrm{e}^{\lambda s} \mathbb{E}\left|f\left(s, X_{s}\right)\right|_{U}^{2} d s \\
& +3 c H(2 H-1) M^{2} t^{2 H-1} \int_{0}^{t} \mathrm{e}^{\lambda s}|g(s)|_{L_{Q}^{0}(K, U)}^{2} d s
\end{aligned}
$$


and, for the chosen parameter $\theta$,

$$
\begin{aligned}
\mathrm{e}^{(\lambda-\theta) t} \mathbb{E}|X(t)|_{U}^{2} \leq & 3 M^{2} \mathrm{e}^{-\theta t} \mathbb{E}|\varphi(0)|_{U}^{2}+\frac{3}{\lambda} M^{2} \mathrm{e}^{-\theta t} \int_{0}^{t} \mathrm{e}^{\lambda s} \mathbb{E}\left|f\left(s, X_{s}\right)\right|_{U}^{2} d s \\
& +3 c H(2 H-1) M^{2} t^{2 H-1} \mathrm{e}^{-\theta t} \int_{0}^{t} \mathrm{e}^{\lambda s}|g(s)|_{L_{Q}^{0}(K, U)}^{2} d s \\
\leq & 3 M^{2} \mathrm{e}^{-\theta t} \mathbb{E}|\varphi(0)|_{U}^{2}+\frac{3}{\lambda} M^{2} \int_{0}^{t} \mathrm{e}^{(\lambda-\theta) s} \mathbb{E}\left|f\left(s, X_{s}\right)\right|_{U}^{2} d s \\
& +3 c H(2 H-1) M^{2} t^{2 H-1} \mathrm{e}^{-\theta t} \int_{0}^{t} \mathrm{e}^{\lambda s}|g(s)|_{L_{Q}^{0}(K, U)}^{2} d s .
\end{aligned}
$$

Firstly, observe that Condition 3 ensures the existence of a positive constant $A_{1}$ such that

$$
3 c H(2 H-1) M^{2} t^{2 H-1} \mathrm{e}^{-\theta t} \int_{0}^{t} \mathrm{e}^{\lambda s}|g(s)|_{L_{Q}^{0}(K, U)}^{2} d s \leq A_{1} \quad \text { for all } t \geq 0,
$$

whence,

$$
\mathrm{e}^{(\lambda-\theta) t} \mathbb{E}|X(t)|_{U}^{2} \leq A_{1}+3 M^{2} \mathbb{E}|\varphi(0)|_{U}^{2}+\frac{3}{\lambda} M^{2} \int_{0}^{t} \mathrm{e}^{(\lambda-\theta) s} \mathbb{E}\left|f\left(s, X_{s}\right)\right|_{U}^{2} d s .
$$

On the other hand, estimating the last term in (5.2) in view of Condition 2, there exists another positive constant $A_{2}$ such that

$$
\begin{aligned}
\int_{0}^{t} \mathrm{e}^{(\lambda-\theta) s} \mathbb{E}\left|f\left(s, X_{s}\right)\right|_{U}^{2} d s \leq & \int_{0}^{t} \mathrm{e}^{(\lambda-\theta) s} \mathbb{E}\left|f\left(s, X_{s}\right)-f(s, 0)+f(s, 0)\right|_{U}^{2} d s \\
\leq & 2 C_{f} \int_{-r}^{0} \mathrm{e}^{(\lambda-\theta) s} \mathbb{E}|\varphi(s)|^{2} d s+2 C_{f} \int_{0}^{t} \mathrm{e}^{(\lambda-\theta) s} \mathbb{E}|X(s)|^{2} d s \\
& +2 \int_{0}^{t} \mathrm{e}^{(\lambda-\theta) s}|f(s, 0)|_{U}^{2} d s \\
\leq & A_{2}+2 C_{f} \int_{-r}^{0} \mathbb{E}|\varphi(s)|^{2} d s+2 C_{f} \int_{0}^{t} \mathrm{e}^{(\lambda-\theta) s} \mathbb{E}|X(s)|^{2} d s
\end{aligned}
$$

Thus, we have

$$
\begin{aligned}
\mathrm{e}^{(\lambda-\theta) t} \mathbb{E}|X(t)|_{U}^{2} \leq & A_{1}+3 M^{2} \lambda^{-1} A_{2}+3 M^{2} \mathbb{E}|\varphi(0)|_{U}^{2}+6 C_{f} M^{2} \lambda^{-1} \int_{-r}^{0} \mathbb{E}|\varphi(s)|^{2} d s \\
& +6 C_{f} M^{2} \lambda^{-1} \int_{0}^{t} \mathrm{e}^{(\lambda-\theta) s} \mathbb{E}|X(s)|^{2} d s \\
= & A_{3}+6 C_{f} M^{2} \lambda^{-1} \int_{0}^{t} \mathrm{e}^{(\lambda-\theta) s} \mathbb{E}|X(s)|^{2} d s
\end{aligned}
$$

where $A_{3}$ is a suitable positive constant. Gronwall's Lemma conduces us to

$$
\mathrm{e}^{(\lambda-\theta) t} \mathbb{E}|X(t)|_{U}^{2} \leq A_{3} \mathrm{e}^{6 C_{f} M^{2} \lambda^{-1} t}
$$

and, consequently,

$$
\begin{aligned}
\mathbb{E}|X(t)|_{U}^{2} & \leq A_{3} \mathrm{e}^{\left(6 C_{f} M^{2} \lambda^{-1}-\lambda+\theta\right) t} \\
& =A_{3} \mathrm{e}^{-\gamma t}
\end{aligned}
$$

The proof is therefore complete. 
Remark 2. The previous theorem states, in particular, that the exponential decay to zero in mean square of the mild solutions to the equation

$$
\left\{\begin{array}{l}
d X(t)=A Y(t) d t+g(t) d B_{Q}^{H}(t), t \geq 0 \\
X(t)=\varphi(t), t \in[-r, 0]
\end{array}\right.
$$

is preserved for our equation (3.1) provided that Conditions 1-3 and (5.1) are satisfied.

Another remarkable fact is that the decay rate $\gamma$ is independent of $H$. Indeed, in case of considering a $Q$-Brownian motion, i.e., the case $H=1 / 2$, instead of our $f B m B_{Q}^{H}$, the condition on $\lambda$ would be exactly (5.1) (to check this assertion, it is enough to take into account the isometry for classical Wiener integrals). In other words, whenever the stochastic integral is well-defined, and under Conditions 1-3, the rate of the exponential decay to zero in mean square is insensitive to the Hurst parameter $H$.

Remark 3. Theorem 3 remains true if we replace the first part of Condition 2 by Condition 4 below.

Condition 4. For any $x \in C(-r, T ; U)$,

$$
\int_{0}^{t} e^{m s}\left|f\left(s, x_{s}\right)\right|_{U}^{2} d s \leq A_{2}+2 C_{f} \int_{-r}^{t} e^{m s}|x(s)|_{U}^{2} d s, \text { for all } 0 \leq m \leq \lambda .
$$

As two canonical applications of our general functional equation (3.1), we will consider the cases of variable and distributed delay. We will formulate that these two situations can be covered by our general functional framework.

5.1. The variable delay case. Let us consider the system

$$
\left\{\begin{array}{l}
d X(t)=(A X(t)+F(t, X(t-\delta(t)))) d t+g(t) d B_{Q}^{H}(t) \\
X(t)=\varphi(t), \quad t \in[-r, 0],
\end{array}\right.
$$

where $r>0$. Let us assume the previous hypotheses on operators $A, g$ and the fractional Brownian motion, and assume now that $F:[0,+\infty) \times U \rightarrow U$ is a measurable function such that

$$
|F(t, x)-F(t, y)|_{U} \leq b_{0}|x-y|_{U}, \quad \text { for all } x, y \in U, \text { and all } t \geq 0,
$$

where $b_{0}$ is a non-negative constant, and

$$
\int_{0}^{\infty} \mathrm{e}^{\lambda s}|F(s, 0)|_{U}^{2} d s<\infty .
$$

For the delay function $\delta$, we assume that $\delta:[0,+\infty) \rightarrow[0, r]$ is differentiable, and there exists a positive $\delta^{*}$ such that

$$
\left|\frac{1}{1-\delta^{\prime}(t)}\right| \leq \delta^{*}, \quad \text { for all } t \geq 0
$$

Observe that our new problem (5.3) can be re-written in our abstract functional formulation by defining a new function $f:[0,+\infty) \times C(-r, 0 ; U) \rightarrow U$ as follows

$$
f(t, \xi)=F(t, \xi(-\delta(t))), \quad \text { for } \xi \in C(-r, 0 ; U), \text { and } t \geq 0 .
$$

We can now establish the following result on the asymptotic behavior of the solutions to problem (5.3). 
Theorem 4. In addition to Conditions 1 and 3, (5.4), (5.5) and (5.6), assume that the mild solution $X(t)$ to the system (5.3), corresponding to the initial function $\varphi \in C\left(-r, 0 ; L^{2}(\Omega ; U)\right)$, exists for all $t \geq-r$, and that

$$
\lambda^{2}>6 M^{2} \delta^{*} b_{0}^{2} e^{\lambda r}
$$

Then, there exists a constant $\gamma>0$ such that

$$
\limsup _{t \rightarrow \infty}\left(\frac{1}{t}\right) \log \mathbb{E}|X(t)|_{U}^{2} \leq-\gamma .
$$

In other words, every mild solution exponentially decays to zero in mean square.

Proof. To prove this result, we will check that the assumptions in Theorem 3 are fulfilled, and for that we only need to check Condition 2 and (5.1).

First, observe that the function $\rho(s)=s-\delta(s)$ is differentiable and, due to the assumptions on $\delta$, is also invertible and satisfies

$$
\rho^{-1}(\sigma) \leq \sigma+r, \quad \text { for all } \sigma \geq-r .
$$

Then, for $0 \leq m \leq \lambda$, by performing the change of variable $\sigma=s-\delta(s)$ in the integral, we obtain

$$
\begin{aligned}
\int_{0}^{t} \mathrm{e}^{m s}\left|f\left(s, x_{s}\right)-f\left(s, y_{s}\right)\right|_{U}^{2} d s & =\int_{0}^{t} \mathrm{e}^{m s}|F(s, x(s-\delta(s)))-F(s, y(s-\delta(s)))|_{U}^{2} d s \\
& \leq b_{0}^{2} \int_{0}^{t} \mathrm{e}^{m s}|x(s-\delta(s))-y(s-\delta(s))|_{U}^{2} d s \\
& \leq b_{0}^{2} \delta^{*} \int_{-r}^{t-\delta(t)} \mathrm{e}^{m \rho^{-1}(\sigma)}|x(\sigma)-y(\sigma)|_{U}^{2} d \sigma \\
& \leq b_{0}^{2} \delta^{*} \mathrm{e}^{m r} \int_{-r}^{t} \mathrm{e}^{m \sigma}|x(\sigma)-y(\sigma)|_{U}^{2} d \sigma \\
& \leq b_{0}^{2} \delta^{*} \mathrm{e}^{\lambda r} \int_{-r}^{t} \mathrm{e}^{m \sigma}|x(\sigma)-y(\sigma)|_{U}^{2} d \sigma,
\end{aligned}
$$

and, therefore, Condition 2 and assumption (5.1) are fulfilled by taking $C_{f}=$ $b_{0}^{2} \delta^{*} \mathrm{e}^{\lambda r}$.

As a specific application of this variable delay case we can consider the following example.

Let $K=L^{2}(0, \pi)$ and $e_{n}=\sqrt{\frac{2}{\pi}} \sin (n x), n \in \mathbb{N}$. Then $\left\{e_{n}\right\}_{n \in \mathbb{N}}$ is a complete orthonormal basis in $K$. Let $U=L^{2}(0, \pi)$ and $A=\frac{\partial^{2}}{\partial x^{2}}$ with domain $D(A)=$ $H_{0}^{1}(0, \pi) \cap H^{2}(0, \pi)$. Then, it is well-known that $A u \stackrel{\partial x^{2}}{=}-\sum_{n=1}^{\infty} n^{2}\left\langle u, e_{n}\right\rangle e_{n}$ for any $u \in U$, and $A$ is the infinitesimal generator of a strongly continuous semigroup of bounded linear operators $S(t): U \rightarrow U$, where $S(t) u=\sum_{n=1}^{\infty} \exp \left(-n^{2} t\right)\left\langle u, e_{n}\right\rangle e_{n}$. In order to define the operator $Q: K \rightarrow K$, we choose a sequence $\left\{\sigma_{n}\right\}_{n \geq 1} \subset \mathbb{R}^{+}$ and set $Q e_{n}=\sigma_{n} e_{n}$, and assume that $\operatorname{tr}(Q)=\sum_{n=1}^{\infty} \sqrt{\sigma}_{n}<\infty$. Define the process $B_{Q}^{H}(s)$ by

$$
B_{Q}^{H}(t)=\sum_{n=1}^{\infty} \sqrt{\sigma_{n}} \beta_{n}^{H}(t) e_{n}
$$


where $H \in(1 / 2,1)$ and $\left\{\beta_{n}^{H}\right\}_{n \in \mathbb{N}}$ is a sequence of two-sided one-dimensional fractional Brownian motions mutually independent.

Then we consider the following stochastic evolution equation:

$$
\left\{\begin{array}{l}
d X(t)=\left[\frac{\partial^{2}}{\partial x^{2}} X(t)+b(t) \frac{X(t-r(1+\sin t))}{1+(X(t-r(1+\sin t)))^{2}}\right] d t+g(t) d B_{Q}^{H}(t), \\
\xi(t, 0)=\xi(t, \pi)=0, t \geq 0 \\
X(t)=\varphi(t), t \in[-r, 0]
\end{array}\right.
$$

where $r \in(0,1)$, and $b, g: \mathbb{R}^{+} \rightarrow \mathbb{R}$ are continuous functions such that $g$ satisfies Condition 3 above and $b$ satisfies

$$
\int_{0}^{\infty} e^{\lambda s}|b(s)|^{2} d s<\infty .
$$

Observe that the fact $\int_{0}^{\infty} e^{\lambda s}|b(s)|^{2} d s<\infty$ implies that $b(t)$ is bounded for all $t \geq 0$. Denote by $b_{0}$ the smallest upper bound of the function $b$.

Then, it is straightforward to check that there exists a unique mild solution to (5.7).

If we assume, in addition, that

$$
1>\frac{6 b_{0} \mathrm{e}^{r}}{1-r}
$$

then, any mild solution to (5.7) decays exponentially to zero in mean square.

Indeed, observe that in our situation it is easy to check that $F(t, x)=b(t) \frac{x}{1+x^{2}}$ is globally Lipschitz with respect to its second variable (i.e. (5.4) is fulfilled), and we have that

$$
M=1, \lambda=1 \text { and } \frac{1}{|1-r \cos t|} \leq \delta^{*}=\frac{1}{1-r} \text { for } t \geq 0 .
$$

Consequently, all the hypotheses in Theorem 4 are satisfied and we can ensure the exponential asymptotical decay to zero in mean square of any mild solution of the system.

5.2. The distributed delay case. Let us now consider the system

$$
\left\{\begin{array}{l}
d X(t)=\left(A X(t)+\int_{-r}^{0} F(t, s, X(t+s)) d s\right) d t+g(t) d B_{Q}^{H}(t) \\
X(t)=\varphi(t), \quad t \in[-r, 0],
\end{array}\right.
$$

where $r>0$. Let us assume the same hypotheses on operators $A, g$ and the fractional Brownian motion as in the variable delay case, and assume now that $F:[0,+\infty) \times[-r, 0] \times U \rightarrow U$ is a measurable function such that

$$
|F(t, s, x)-F(t, s, y)|_{U} \leq b_{0}|x-y|_{U}, \forall x, y \in U, t \geq 0, s \in[-r, 0],
$$

where $b_{0}$ is a non-negative constant, and

$$
\int_{0}^{\infty} \mathrm{e}^{\lambda s}\left(\int_{-r}^{0}|F(s, \sigma, 0)|_{U}^{2} d \sigma\right) d s<\infty
$$

Then, we can set this problem in our abstract formulation by writing

$$
f(t, \xi)=\int_{-r}^{0} F(t, s, \xi(s)) d s, \text { for } \xi \in C(-r, 0 ; U),
$$


and we formulate the following result for problem (5.8).

Theorem 5. In addition to Conditions 1 and 3, (5.9) and (5.10), assume that the mild solution $X(t)$ to the system (5.8), corresponding to the initial function $\varphi \in C\left(-r, 0 ; L^{2}(\Omega ; U)\right)$, exists for all $t \geq-r$, and that

$$
\lambda^{3}>6 M^{2} r b_{0}^{2}\left(e^{\lambda r}-1\right) .
$$

Then, there exists a constant $\gamma>0$ such that

$$
\limsup _{t \rightarrow \infty}\left(\frac{1}{t}\right) \log \mathbb{E}|X(t)|_{U}^{2} \leq-\gamma .
$$

In other words, every mild solution exponentially decays to zero in mean square.

Proof. The proof follows by simply checking that the assumptions in Theorem 3 hold. It is not difficult to prove that the constant $C_{f}$ is given by

$$
C_{f}=\left(\mathrm{e}^{\lambda r}-1\right) r b_{0}^{2} \lambda^{-1},
$$

whence the conclusion of the theorem follows immediately. We leave the details to the reader.

\section{Acknowledgments}

The authors would like to thank the anonymous referee for the interesting remarks and comments which helped to improve the paper.

Takeshi Taniguchi is partly supported by the Grant-in-Aid for Scientific Research (19510164) from Japan Society for the Promotion of Science (JSPS), Japan, and by the Grant-in-Aid for Academic Research from the ISHIBASHI Foundation, Japan. Tomás Caraballo and María J. Garrido-Atienza are partly supported by Ministerio de Ciencia e Innovación (Spain) under the grant MTM2008-00088, and by Junta de Andalucía (Spain) under Proyecto de Excelencia P07-FQM-02468.

\section{REFERENCES}

[1] E. Alos, O. Mazet and D. Nualart. Stochastic calculus with respect to Gaussian processes. Ann. Probab., 29 (1999), 766-801.

[2] B. Boufoussi and S. Hajji. Functional differential equations driven by a fractional Brownian motion, submitted (2009).

[3] G. Da Prato and J. Zabczyk, Stochastic Equations in Infinite Dimensions, Cambridge University Press, 1992.

[4] M. Ferrante and C. Rovira. Stochastic delay differential equations driven by fractional Brownian motion with Hurst parameter $H>1 / 2$. Bernouilli, 12 (2006), 85-100.

[5] M. Ferrante and C. Rovira. Convergence of delay differential equations driven by fractional Brownian motion, Journal of Evolution Equations, 10 (2010), no. 4, 761-783.

[6] M.J. Garrido-Atienza, K. Lu and B. Schmalfuss. Random dynamical systems for stochastic partial differential equations driven by a fractional Brownian motion, Discrete and Continuous Dynamical Systems, Series B, 14 (2010), no. 2, 473-493.

[7] M.J. Garrido-Atienza, B. Maslowski and B. Schmalfuss. Random attractors for stochastic equations driven by a fractional Brownian motion. International Journal of Bifurcation and Chaos, 20 (2010), no. 9, 2761-2782.

[8] W. Grecksch and V.V. Anh. A parabolic stochastic differential equation with fractional Brownian motion input. Statist. Probab. Lett., 41 (1999), 337-345.

[9] M. Gubinelli, A. Lejay and S. Tindel. Young integrals and SPDEs. Potential Anal. An International Journal Devoted to the Interactions between Potential Theory, Probability Theory, Geometry and Functional Analysis, 25 (2006), no. 4, 307-326.

[10] Y. Kuang. Delay Differential Equations with Applications in Population Dynamics, Academic Press, Boston, 1993. 
[11] J. K. Hale and S.M. Verduyn Lunel. Introduction to Functional Differential Equations, Springer, New York, 1995.

[12] A. Manitius. Feedback controllers for a wind tunnel model involving a delay: Analytical design and numerical simulation. IEEE Transactions on Authomatic Control, 29 (1984), no. $12,1058-1068$.

[13] B. Maslowski and D. Nualart. Evolution equations driven by a fractional Brownian motion. J. Funct. Anal., 202 (2003), no. 1, 277-305.

[14] Y. Mishura. Stochastic Calculus for Fractional Brownian Motion and Related Topics, Lecture Notes in Mathematics 1929, 2008.

[15] J.D. Murray. Mathematical Biology, Springer-Verlag, Berlin, 1993.

[16] A. Neuenkirch, I. Nourdin and S. Tindel. Delay Equations driven by Rough Paths. Elec. J. Probab., 13 (2008), 2031-2068.

[17] D. Nualart. The Malliavin Calculus and Related Topics, Second Edition, Springer-Verlag, Berlin, 2006.

[18] D. Nualart and A. Răşcanu. Differential equations driven by fractional Brownian motion. Collect. Math., 53 (2002), no 1, 55-81.

[19] S. Tindel, C. Tudor and F. Viens. Stochastic Evolution Equations with Fractional Brownian Motion. Probability Theory and Related Fields, 127 (2003), no. 2, 186-204.

(T. Caraballo) Dpto. Ecuaciones Diferenciales y AnÁlisis Numérico, Universidad de Sevilla, Apdo. de Correos 1160, 41080-Sevilla (Spain),

E-mail address, T. Caraballo: caraball@us.es

(M. J. Garrido-Atienza) Dpto. Ecuaciones Diferenciales y AnÁlisis Numérico, Universidad de Sevilla, Apdo. de Correos 1160, 41080-Sevilla (Spain),

E-mail address, M. J. Garrido-Atienza: mgarrido@us.es

(T. Taniguchi) Division of Mathematical Sciences, Graduate School of Comparative Culture, Kurume University, Miımachi, Kurume, Fukuoka 839-8502 (Japan),

E-mail address, T. Taniguchi: takeshi_taniguchi@kurume-u.ac.jp 